This is the peer reviewed version of the following article:

Stojanović, Zoran, Mojca Otoničar, Jongwook Lee, Magdalena M. Stevanović, Mintai P. Hwang, Kwan Hyi Lee, Jonghoon Choi, and Dragan Uskoković. 2013. "The Solvothermal Synthesis of Magnetic Iron Oxide Nanocrystals and the Preparation of Hybrid Poly(l-lactide)-polyethyleneimine Magnetic Particles." Colloids and Surfaces B: Biointerfaces 109 (September 1): 236-243.

http://dx.doi.org/10.1016/j.colsurfb.2013.03.053.

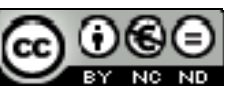

This work is licensed under a Creative Commons - Attribution-Noncommercial-No Derivative Works 3.0 Serbia. 


\title{
The solvothermal synthesis of magnetic iron oxide nanocrystals and the preparation of hybrid poly(L-lactide)-polyethyleneimine magnetic particles
}

Zoran Stojanović ${ }^{\dagger}$, Mojca Otoničar ${ }^{\S}$, Jongwook Lee ${ }^{\ddagger}$, Magdalena M. Stevanović ${ }^{\dagger}$, Mintai P. Hwang ${ }^{\ddagger}$, Kwan Hyi Lee $^{\ddagger}$, Jonghoon Choi ${ }^{\ddagger}$, Dragan Uskoković ${ }^{\dagger}$

${ }^{\dagger}$ Centre for Fine Particles Processing and Nanotechnologies, Institute of Technical Sciences of SASA, Knez Mihailova 35, Belgrade, Republic of Serbia

$\S$ Department of Advanced Materials K9, Jožef Stefan Institute, Jamova cesta 39, Ljubljana, Republic of Slovenia

* Center for Biomaterials, Biomedical Research Institute, Korea Institute of Science and Technology, Hwarangno 14-gil 5, Seongbuk-gu, 136-791, Seoul, Republic of Korea

\begin{abstract}
We report a simple and green procedure for the preparation of magnetic iron oxide nanocrystals via solvothermal synthesis. The nanocrystal synthesis was carried out under mild conditions in the water-ethanololeic acid solvent system with the use of the oleate anion as a surface modifier of nanocrystals and glucose as a reducing agent. Specific conditions for homogenous precipitation achieved in such a reaction system lead to the formation of uniform high-quality nanocrystals down to $5 \mathrm{~nm}$ in diameter. The obtained hydrophobic nanocrystals can easily be converted to hydrophilic magnetic nanoparticles by being immobilized in a poly(Llactide) - polyethyleneimine polymeric matrix. These hybrid nano-constructs may find various biomedical applications, such as magnetic separation, gene transfection and/or magnetic resonance imaging.
\end{abstract}

\section{Keywords}

Magnetic iron oxides, Nanocrystals, Solvothermal synthesis, Green synthesis route, Surface functionalization, Oleic acid, Polyethyleneimine, Poly (L-lactide)

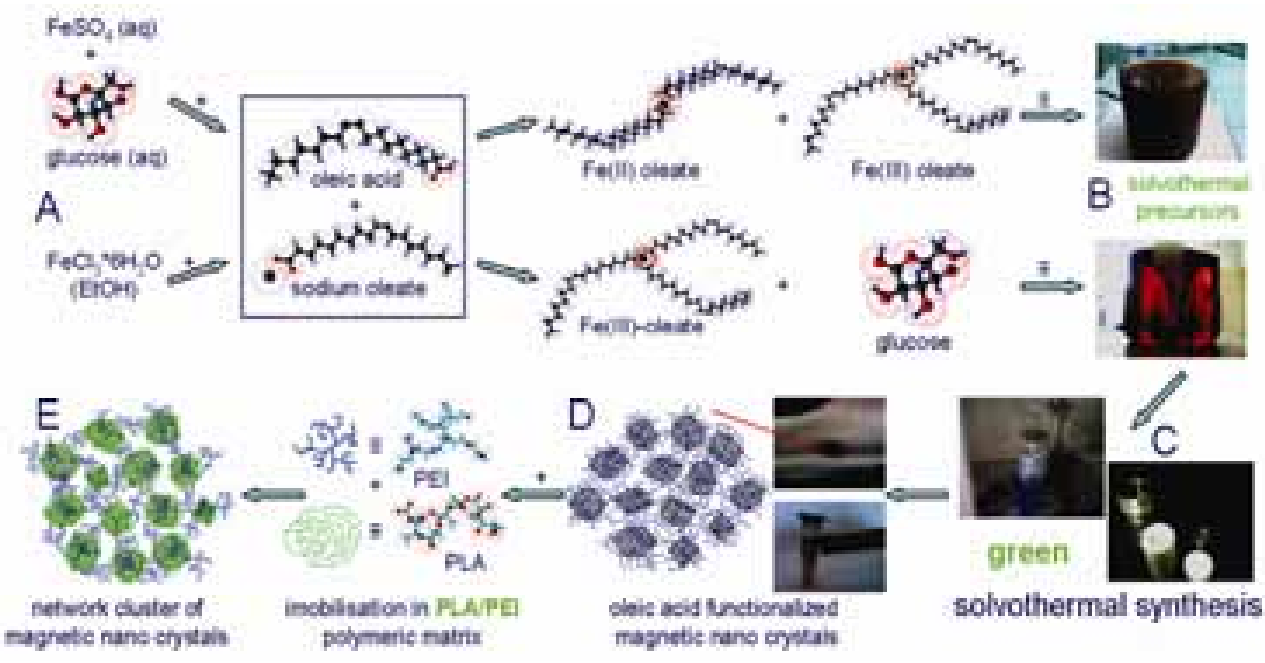




\section{Introduction}

The high magnetic susceptibility and superparamagnetism found in iron oxide nanoparticles of magnetite $\left(\mathrm{Fe}_{3} \mathrm{O}_{4}\right)$ and its oxidized form, maghemite $\gamma\left(\mathrm{Fe}_{2} \mathrm{O}_{3}\right)$, have propelled their use in various technological fields, e.g. molecular and cellular magnetic resonance imaging [1], magnetic force-assisted delivery [2], various separation techniques of cells, proteins, viruses, etc. [3, 4, 5], AC magnetic field cancer treatment [6], data storage [7], catalysis and adsorbent recovery [8,9], magnetorheological shock absorbers and seals $[10,11]$ are only some of its applications in the biomedical and technical fields.

The physical properties of nanocrystals, and those of iron oxide in particular, are strongly related to their size and shape. In other words, the production of monodisperse high-quality nanocrystals is crucial for scientific research and applications $[12,13]$. Advances made in high-temperature reactions using organic solutions have led to the production of high-quality monodisperse superparamagnetic iron oxide nanoparticles smaller than $20 \mathrm{~nm}[14,15]$. Although methods based on aqueous solution co-precipitation are advantageous; they confer hydrophilicity to the synthesized nanoparticles; they are non-toxic, cost-effective, and energyefficient; but they still do not yield high-quality nanocrystals [16]. The microemulsion method is another approach that has shown to be efficient in the synthesis of a wide variety of magnetic nanoparticles but one that is hampered with relatively high polydispresity, and inefficient and non-economic synthesis [17].

Hydrothermal and solvothermal synthesis approaches are based on chemical reactions at elevated temperatures and pressures ranging from ambient temperatures and pressures to above critical conditions. They cover a wide range of chemical and thermodynamic parameters, making them a powerful tool for materials processing in a very broad sense [18, 19]. Recent achievements in the solvothermal synthesis of monodisperse nanocrystals are related to a variety of nanocrystals with hydrophobic properties synthesized using different ligands composed of long aliphatic chains and polar coordinating head groups [20]. Magnetite and other ferrite compounds are among those produced using a similar solvothermal liquid-solid-solution (LSS) strategy [21, 22]. Several groups have reported the synthesis of iron oxide NPs by hydrothermal method. However, despite distinct efforts to find appropriate ligands to synthesize monodisperse nanocrystals in a hydrophilic environment [20], hydrothermal approaches still fail to generate quality nanocrystals smaller than $10 \mathrm{~nm}$ exhibit hydrophilic surface properties, with the exception of some noble metal nanocrystals. For example, Wang et al. [23] have described a one-step hydrothermal process to prepare highly crystalline $\mathrm{Fe}_{3} \mathrm{O}_{4}$ nanopowders without the use of surfactants. The obtained $\mathrm{Fe}_{3} \mathrm{O}_{4}$ particles had the mean size of about $40 \mathrm{~nm}$. Zheng et al. [24] have proposed a hydrothermal route for preparing $\mathrm{Fe}_{3} \mathrm{O}_{4}$ nanoparticles with a diameter of 27 $\mathrm{nm}$ in the presence of a surfactant, sodium bis(2-ethylhexyl)sulfosuccinate. Daou et al. [25] have reported the obtaining of magnetite particles with an average size of $39 \mathrm{~nm}$ by hydrothermal treatment at $250{ }^{\circ} \mathrm{C}$. Recently, Zhu et al [26] have synthesized $\mathrm{Fe}_{3} \mathrm{O}_{4}$ nanoparticles with sizes at around 30-40 nm by a sonochemical method in the presence of graphene oxide.

Water-soluble superparamagnetic magnetite nanoparticles can also be obtained via a simple hydrothermal process using affordable and non-toxic chemicals under relatively mild conditions by the introduction of ligands with bi-functional roles as reducing and capping agents [27, 28, 29]. 
Inspired by LSS, the nanocrystal synthesis strategy [21], and the use of glucose as a reducing agent [30, 31], we have designed and analyzed a simple solvothermal system for the synthesis of magnetic iron oxide nanocrystals which exploits all benefits of traditional solvothermal approaches. A number of researchers successfully synthesized magnetic nanoparticles. However, the green solvothermal synthesis method described in this paper overcomes to a great extent some of the disadvantages previously reported by researchers who used other methods, such as impurities, size and distribution control, economic viability and difficulties during preparation that limit their commercialization potential. It involves affordable and non-toxic chemicals, generates a high-yield of nearly monodisperse nanocrystals, does not require a protective environment, and its reaction solvents may be recycled after the reaction. Here, we report the synthesis of magnetic iron oxides via novel chemical routes enabling us to obtain nanocrystals of magnetite (or maghemite) as small as $5 \mathrm{~nm}$, with a narrow size distribution. A simple and original solvothermal approach to obtaining nanoparticles starting from different valence states of iron $(2+$ or $3+)$ is presented. The initially generated nanocrystals are hydrophobic and convenient for encapsulation within polymeric matrices. Accordingly, one of the major advantages of this processing method is also the ability to change the hydrophobicity to hydrophilicity by choosing different functional groups on the surface of nanoparticles. In this study, we have demonstrated a simple procedure for the immobilization of superparamagnetic nanocrystals in the ambiphilic biodegradable poly(L-lactide) (PLA) polymer containing water dispersible polyethyleneimine (PEI). These hybrid nanostructures could provide a platform for magnetic field-assisted gene transfection [32], multifunctional imaging, and targeted delivery of genes and various hydrophobic active substances.

\section{Materials and Methods}

\subsection{Materials for the preparation of magnetic nanocrystals and hybrid hydrophilic PLA-PEI/magnetic nanoparticles}

Ferrous sulfate heptahydrate $\left(\mathrm{FeSO}_{4} * 9 \mathrm{H}_{2} \mathrm{O}\right.$, Acros Organics, Belgium) and ferric chloride hexahydrate $\left(\mathrm{FeCl}_{3} * 6 \mathrm{H}_{2} \mathrm{O}\right.$, Acros Organics, Belgium) were used. For the preparation of oleate species as functionalizing agents sodium hydroxide $(\mathrm{NaOH}$, Kemika, Croatia), oleic acid (cis-9-octadecenoic acid, Fisher Chemical, Belgium) and oleylamine (cis 1-amino-9-octadecen, Fisher Chemical, Belgum) were used. Ethanol and distilled water were used as solvents. Glucose (anhydrous $\mathrm{C}_{6} \mathrm{H}_{12} \mathrm{O}_{6}$, Zorka Pharma, Serbia) was used as a reducing agent and a stabilizer of the $\mathrm{Fe}(\mathrm{II})$ oxidation state.

For the preparation of hybrid PLA-PEI/MNp, the synthesized hydrophobic nanoparticles were used in conjunction with poly(L-lactide) (PLA, MW $\approx 160000 \mathrm{~g} / \mathrm{mol}$, Lactel Absorbable Polymers, USA), branched polyethyleneimine (PEI, 50\% water solution, MW $\approx 2000 \mathrm{~g} / \mathrm{mol}$, Sigma-Aldrich, USA), and polyvinyl alcohol (PVA, MW $\approx 13000-23000$ g/mol, 87-89 \% hydrolyzed, Sigma-Aldrich, USA). Chloroform was also used as a polymer solvent. 


\subsection{Synthesis of magnetic iron oxide nanocrystals starting from the $\mathrm{Fe}(\mathrm{II})$ precursor}

An amount of $1.05 \mathrm{~g}$ of $\mathrm{NaOH}(\approx 26 \mathrm{mmol})$ was dissolved in $2 \mathrm{ml}$ of distilled water and after an amount of $15 \mathrm{ml}$ of ethanol was added and vigorously mixed. This solution was added to $10.5 \mathrm{ml}$ of oleic acid, which was heated to approximately $70{ }^{\circ} \mathrm{C}$ and continuously mixed to obtain a transparent yellow sodium oleate solution. The preparation of an iron precursor solution involved the dissolution of $0.63 \mathrm{~g}$ of anhydrous glucose in $33 \mathrm{ml}$ of freshly distilled water in a 100-ml Teflon liner and subsequent addition of $1.46 \mathrm{~g}$ of $\mathrm{FeSO}_{4} * 7 \mathrm{H}_{2} \mathrm{O}$ $(\approx 5.2 \mathrm{mmol})$ under continuous stirring. The oleate solution was then added drop-wise into the iron precursor solution. After a few drops, the solution became turbid. Further adding of the oleate solution led to the formation of viscous light olive green suspension, which became less viscous and reddish at the end of the process. The suspension was then stirred for an hour. A liner was capped and sealed in a stainless steel autoclave, immersed in an oil bath at a designated temperature and magnetically stirred during the reaction.

After the reaction had been completed, the autoclave was submerged into cold water. The liquid phase was decanted and discarded, and the black precipitate on the magnetic bar was separated and washed in ethanol several times and collected with a magnet. Particles were dried in air at $90{ }^{\circ} \mathrm{C}$ for three hours. The yields of these reactions were around $200 \mathrm{mg}$ of washed and dried particles, or approximately $50 \%$, compared to the theoretical yield of $\mathrm{Fe}_{3} \mathrm{O}_{4}$ or $\gamma-\mathrm{Fe}_{2} \mathrm{O}_{3}$, not taking into account the organic coating on particles. A summary of reaction parameters is given in Table 1, samples are denoted as $\mathrm{S} 1$ to $\mathrm{S} 3$.

\subsection{Synthesis of magnetic iron oxide nanocrystals starting from the $\mathrm{Fe}(\mathrm{III})$ precursor}

To prepare magnetic iron oxide nanocrystals starting from the Fe(III) precursor, $1.765 \mathrm{~g}$ of oleylamine $(\approx 6.6 \mathrm{mmol})$ and $5.5 \mathrm{ml}$ of oleic acid $(\approx 17 \mathrm{mmol})$ were mixed with $33 \mathrm{ml}$ of ethanol. An amount of $0.5946 \mathrm{~g}$ of $\mathrm{FeCl}_{3} * 6 \mathrm{H}_{2} \mathrm{O}(\approx 2.2 \mathrm{mmol})$ was added to this solution and mixed additionally for 30 minutes at $50{ }^{\circ} \mathrm{C}$. The solution was then transferred into a Teflon liner and continuously stirred. A volume of $11 \mathrm{ml}$ of an aqueous solution of $\mathrm{NaOH}$ and glucose was added and stirred for another $30 \mathrm{~min}$. A magnetic stirring bar was not used during the solvothermal treatment to avoid the influence of a magnetic field during synthesis. The liner was then capped and sealed in a stainless steel autoclave and immersed into a preheated oil bath.

After the reaction had been completed, the autoclave was submerged into cold water to cool. The liquid phase was decanted and precipitates were washed in ethanol several times and collected with a magnet. Particles were dried in air for three hours at $90{ }^{\circ} \mathrm{C}$. The yield of the precipitated particles increased with the amount of $\mathrm{NaOH}$ added and it was approximately $50 \%$ or less. The summary of the reaction parameters for the preparation of these samples, denoted as S4 to S6, is given in Table 1. 


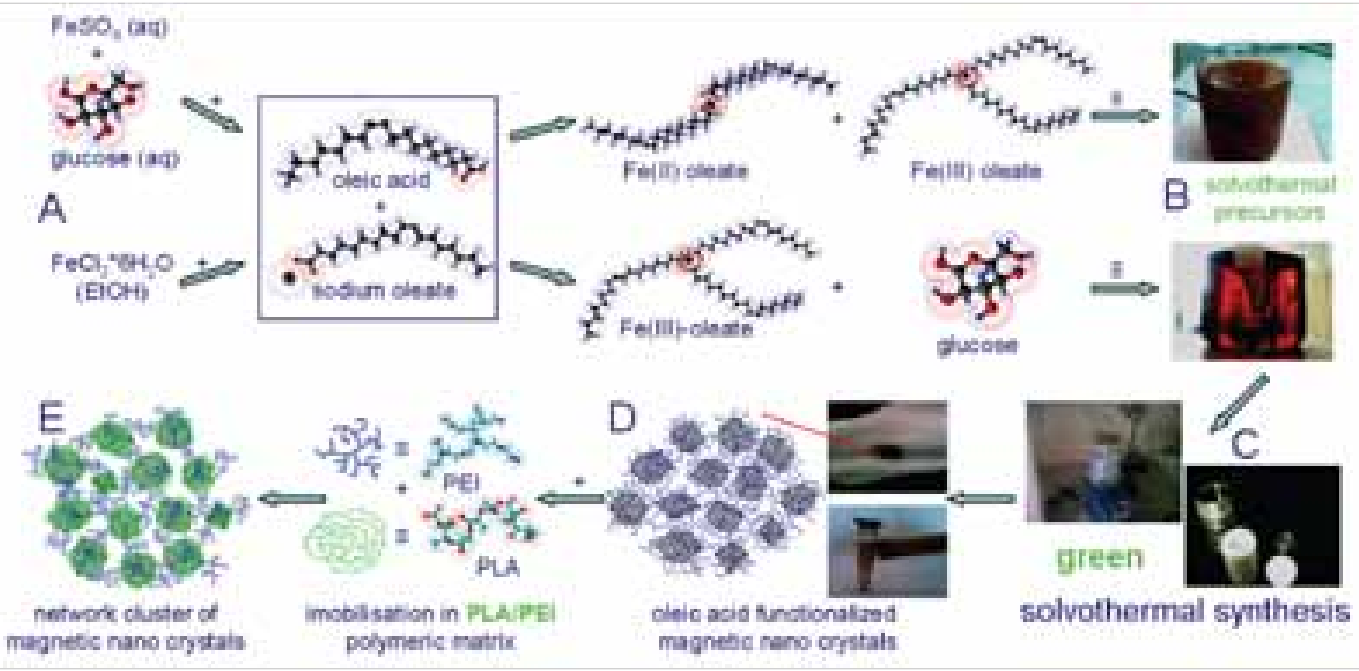

Figure 1. Procedures for the preparation of iron oxide magnetic nanocrystals starting from two different precursor salts (A), appearance of solvothermal precursors (B), $100 \mathrm{ml}$ autoclave and the heating set-up used for solvothermal synthesis (C), nanocrystals functionalized with oleic acid, images of dry powder and nanocrystals re-dispersed in chloroform attracted by magnet (D). The magnetic nanocrystals are arranged in 3D network clusters (E).

Then the experiments were carried out with a modified protocol. Only oleic acid, sodium hydroxide and glucose were used in the reaction (oleylamine was not used). Glucose was used as a reducing agent. After the reaction had been completed, the autoclave was pulled out from the oil bath and immersed into cold water to cool. The liquid phase from the liner was decanted and the liner was washed with $4 \mathrm{ml}$ of cyclohexane to remove precipitates from its bottom. The particles dispersed in cyclohexane were poured into a glass beaker and an amount of about $20 \mathrm{ml}$ of ethanol was added to re-precipitate the particles. After sedimentation, the liquid phase was decanted and the procedure was repeated. The precipitate was dried in air at $50{ }^{\circ} \mathrm{C}$ for two hours. The yield of particles measured after drying was high, around 100\%, not taking into account the organic phase. The reaction parameters for these samples, labeled as S7 to S9, are given in Table 1.

Table 1. The synthesis parameters for the samples synthesized from the Fe(II) precursor salt and Fe(III) precursor salt

\begin{tabular}{|c|c|c|c|c|c|c|c|c|c|c|}
\hline Label of sample & $\begin{array}{c}\mathrm{n}_{\mathrm{Fe}}^{2+} \\
(\mathrm{mmol})\end{array}$ & $\begin{array}{c}\mathrm{n}_{\mathrm{Fe}}^{3+} \\
(\mathrm{mmol})\end{array}$ & $\begin{array}{l}\text { Oleylamine } \\
(\mathrm{mmol})\end{array}$ & $\begin{array}{c}\mathrm{n}_{\mathrm{NaOH}} \\
(\mathrm{mmol})\end{array}$ & $\mathrm{V}_{\mathrm{H} 2 \mathrm{O}}(\mathrm{ml})$ & $\mathrm{V}_{\mathrm{EtOH}}(\mathrm{ml})$ & $\mathrm{V}_{\mathrm{OlAc}}(\mathrm{ml})$ & $\begin{array}{l}\mathrm{n}_{\text {glucose }} \\
(\mathrm{mmol})\end{array}$ & $\begin{array}{c}\text { Temperature } \\
\left({ }^{\circ} \mathrm{C}\right)\end{array}$ & Reaction time (h) \\
\hline S1 & 5.25 & - & - & 26 & 35 & 15 & 10.5 & 3.5 & 140 & 5 \\
\hline S3 & 5.25 & - & - & 26 & 35 & 15 & 10.5 & 3.5 & 140 & 10 \\
\hline S4 & - & 2.2 & 6.6 & 6.6 & 11 & 33 & 5.5 & 3.9 & 120 & 20 \\
\hline S6 & - & 2.2 & 6.6 & 20 & 11 & 33 & 5.5 & 0.73 & 120 & 20 \\
\hline S7 & - & 5.25 & - & 48 & 4 & 25 & 20 & 1.75 & 150 & 20 \\
\hline S8 & - & 2.64 & - & 52.5 & 14 & 27.5 & 22 & 1.75 & 150 & 20 \\
\hline S9 & - & 2.64 & - & 45.75 & 10 & 30 & 19 & 0.875 & 150 & 20 \\
\hline
\end{tabular}

Each experimental set was repeated minimum three times. 


\subsection{Preparation of hydrophilic PLA-PEI/MNp nanoparticles}

An amount of $60 \mathrm{mg}$ of poly(L-lactide) was dissolved in $1 \mathrm{ml}$ of chloroform, then nanocrystals (60 mg) were added to this solution and dispersed in an ultrasonic bath for 20 seconds. This mixture was added dropwise into a sonicated water-ethanol solution $\left(20 \mathrm{ml} \mathrm{H}_{2} \mathrm{O}\right.$ and $20 \mathrm{ml}$ of ethanol) in which $50 \mathrm{mg}$ of PEI were dissolved. The resulting suspension was poured onto a watch glass and left to dry in air for one day at $40{ }^{\circ} \mathrm{C}$. The dry residue was dissolved in $2 \mathrm{ml}$ of chloroform into which $5 \mathrm{ml}$ of ethanol was added. This dispersion was added drop-wise into a sonicated water-ethanol solution $\left(20 \mathrm{ml}\right.$ of $\mathrm{H}_{2} \mathrm{O}$ and $10 \mathrm{ml}$ of ethanol) in which $20 \mathrm{mg}$ of PVA were dissolved. After the chloroform-ethanol dispersion had been added, the suspension was sonicated for another $10 \mathrm{~min}$. The dispersion of nanocrystals coated with polymers was then poured onto a watch glass, exposed to air flow at $40{ }^{\circ} \mathrm{C}$ for three hours, and filtered through fast filter paper. The obtained water filtrate was poured into a vial and stored at $4{ }^{\circ} \mathrm{C}$ for characterization.

\subsection{Instruments and Measurements}

The identification of crystal phases of dry powders was determined by X-ray powder diffraction (Philips

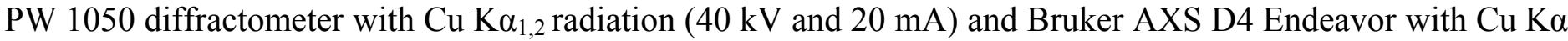
(50 kV and $50 \mathrm{~mA}$ ). The profiles were fitted using Fourier filtering by Gaussians in the entire measuring range by PowderX software (C. Dong, IP CAS, China). The average crystallite size was determined from FWHM of the strongest (311) reflection with Sherrer's equation using the value of 0.89 for Sherrer's constant.

The particle sizes of the synthesized hydrophobic nanocrystals dispersed in chloroform were measured using the dynamic light scattering method (DLS) by a Zetasizer Nano, ZS (Malvern Instruments, UK) with a $633 \mathrm{~nm} \mathrm{He}-\mathrm{Ne}$ laser. The measurement range of this instrument is from $0.3 \mathrm{~nm}$ to 10.0 microns.

The size distribution of hybrid PLA-PEI/MNp in water after one-month storage at $4{ }^{\circ} \mathrm{C}$ was measured on a Malvern Mastersizer 2000 instrument. The size measurement range of this instrument is from $20 \mathrm{~nm}$ up to $2 \mathrm{~mm}$. Prior to measuring, the sample was treated for $30 \mathrm{~min}$ in an ultrasonic bath.

FTIR measurements were performed on an MIDAC M 2000 Series Research Laboratory FTIR Spectrometer using the $\mathrm{KBr}$ pellet technique, in the spectral range of $400-4000 \mathrm{~cm}^{-1}$. The spectral resolution was $4 \mathrm{~cm}^{-1}$.

For an analysis by transmission electron microscopy (TEM), the powders were dispersed in n-hexan in an ultrasonic bath for 20 minutes and then dripped onto a lacey carbon film supported by a 300-mesh copper grid. A TEM 2100 microscope (Jeol Ltd., Tokyo, Japan) operated at $200 \mathrm{kV}$ was used to determine the size and distribution of the nanocrystals, their shape and crystallinity. Selected-area electron diffraction (SAED) analyses were also performed to identify the crystal structure of the particles. The image analysis was carried out using Particle Size Analyzer macro (R. Sperling, ICN, Spain) for ImageJ software (W. Rasband, NIH, US).

Magnetic measurements of dry powder samples were performed on a vibrating sample magnetometer Lake Shore 7307 at room temperature. The magnetization values were normalized to the mass of magnetic material. 


\section{Results and discussion}
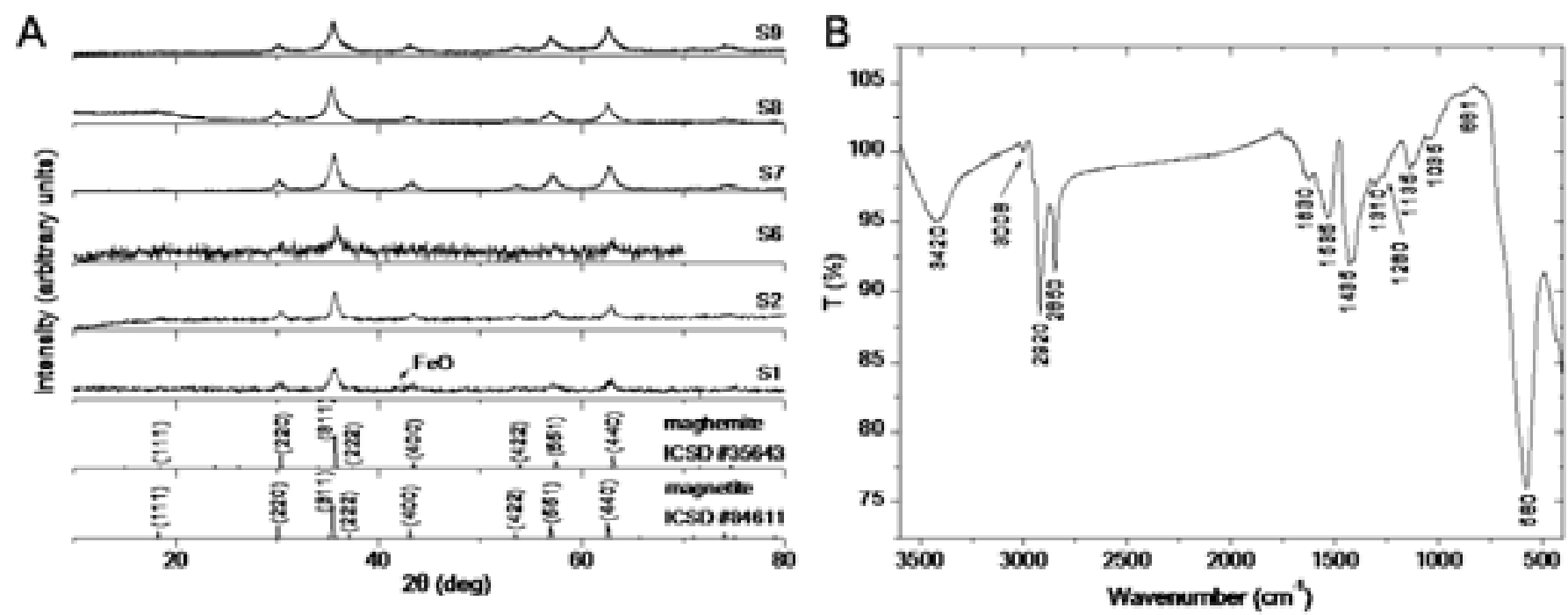

Figure 2. XRD patterns of some samples listed in Table 1 and FTIR spectra of sample S1.

Both protocols for the fabrication of magnetic nanocrystals, from the Fe(II) precursor, as well as from $\mathrm{Fe}(\mathrm{III})$ precursor, are shown schematically in Figure 1. The starting difference between the two protocols lies in the choice of the salt used as a source of iron ions. When iron ion containing solutions were mixed with sodium oleate dissolved in water - ethanol mixture, an iron oleate complex was formed by the ion exchange process. In case when the Fe(II) salt was used, a mixture of the Fe(II) and Fe(III) complex was formed, as a consequence of the partial oxidation of the $\mathrm{Fe}(\mathrm{II})$ species during the operations conducted in air without a protective atmosphere $[33,34]$. This was observed by the color change from olive green to reddish during the preparation of the solvothermal precursor. In the case when the Fe(III) salt was used, the Fe(III) oleate complex was formed by ion exchange. It could be visually detected about 10 minutes after the reaction as red droplets formed in the water-ethanol solvent mixture. When stirring was stopped, these droplets tended to fall on the bottom of the vessel and to stick to the walls. Accordingly, when droplets were formed, stirring was necessary to prevent coalescence and precipitation of droplets and preserve the active surface for the nucleation reaction and, subsequently, for the growth of nanocrystals. In the case when oleic acid was used in excess, e.g. for the sample S9, the Fe(III) oleate complex gets dissolved in such a mixture of water - ethanol - oleic acid. This observation can be linked to solubilization of e.g. calcium soaps in fatty acids [35]. In other words, a homogenous solutionlike reaction mixture can be obtained at a certain ratio of the components, while all other ratios yield two phases. With a homogenous reaction precursor, it is possible to accomplish homogenous precipitation at a certain temperature by the solvolysis of the Fe(III) complex which will eventually lead to the formation of large amount of nuclei instantaneously. The nucleation process can be accompanied or followed by synergetic reduction with glucose and ethanol.

All of the samples listed in Table 1 were characterized by means of XRD, where magnetic iron oxide phases (i.e. magnetite and/or maghemite) were detected in all samples. The XRD patterns of typical samples are shown in Figure $2 \mathrm{~A}$. The only impurity is $\mathrm{FeO}$ in the sample $\mathrm{S} 1$, prepared from the Fe(II) precursor. FeO most likely comes from a large excess of glucose, which retains the Fe(II) oxidative state of ions by reduction and/or 
stabilization in gluconate complex. It is formed due to the dehydration of $\mathrm{Fe}(\mathrm{OH})_{2}$ and/or reduction of $\mathrm{FeO}(\mathrm{OH})$. As a side note, the samples synthesized from the Fe(II) precursor salt in an excess of glucose were magnetically separated from the remaining precipitate, leading us to believe that the amount of $\mathrm{FeO}$ formed in our case was even greater.

It can be concluded from the XRD phase analysis of the samples S6, S7, S8 and S9 that the stoichiometric amount of glucose was sufficient for the reduction of one third of Fe(III) ions for the formation of magnetic iron oxide. These samples were collected from the bottom of the liner and they were washed and re-precipitated several times without any magnetic or centrifugal separation. At certain reactant ratios, larger crystals of $\mathrm{NaCl}$ precipitated as another phase, but for the purpose of this study, these samples were not taken into consideration.

To verify the nature of surface functionalization of magnetic nanocrystals, the FTIR spectra of the sample $\mathrm{S} 1$ have been investigated. The spectrum, as shown in figure $2 \mathrm{~B}$, shows a band at $580 \mathrm{~cm}^{-1}$ characteristic for the $\mathrm{Fe}-\mathrm{O}$ bond vibration in the crystal lattice of iron oxide [36]. The band at $3420 \mathrm{~cm}^{-1}$ is characteristic for vibrations of $\mathrm{O}-\mathrm{H}$ groups and can be attributed to adsorbed ethanol molecules. The bands at $1630 \mathrm{~cm}^{-1}$ can be also be assigned to $\mathrm{O}-\mathrm{H}$ bonds from ethanol molecules. The bands at $881 \mathrm{~cm}^{-1}, 1035 \mathrm{~cm}^{-1}$, $1260 \mathrm{~cm}^{-1}$ and $1310 \mathrm{~cm}^{-1}$ can be attributed to $\mathrm{C}-\mathrm{O}$ bonds from adsorbed $\mathrm{CO}_{2}$ or ethanol. A relatively intense band at $1135 \mathrm{~cm}^{-1}$ indicates the presence of the asymmetric stretch of $\mathrm{C}-\mathrm{O}-\mathrm{C}$ group, which can be due to the cyclic form of glucose [14, 37].

The absence of an intense peak at $1710 \mathrm{~cm}^{-1}$, characteristic for the $\mathrm{C}=\mathrm{O}$ bond stretch in carboxylic groups of liquid oleic acid, the presence of peaks at $2920 \mathrm{~cm}^{-1}$ and $2850 \mathrm{~cm}^{-1}$, characteristic bands for asymmetric and symmetric stretching of the C-H bonds in alkyl chains, and the presence of a small peak at $3008 \mathrm{~cm}^{-1}$, characteristic for the cis $-\mathrm{CH}=\mathrm{CH}-$ conformation of oleic acid, suggest that oleic acid is chemisorbed onto the surface [38]. The presence of two bands in spectra at $1535 \mathrm{~cm}^{-1}$ and $1435 \mathrm{~cm}^{-1} \mathrm{can}$ be attributed to asymmetric and symmetric vibrations of carboxylate groups. The position and difference of these two bands can be used to determine the carboxylate coordination mode. The difference between these two bands is $\Delta=100 \mathrm{~cm}^{-1}$, suggesting a chelating bidentate type of bonding between the carboxylate groups and the surface of the particle, whereas the interaction between the $\mathrm{COO}^{-}$groups and $\mathrm{Fe}$ atoms is covalent in nature [39].

The highly crystalline nature of particles can be observed from TEM images and electron diffraction images presented in Figure 3. The diffraction rings are indexed to the cubic crystal symmetry, and they correspond to magnetite or maghemite, as further confirmed by the XRD analysis.

Some samples were not properly prepared for a TEM analysis due to their agglomeration caused by the lack of re-functionalization after washing. This was not the case with the sample S1, which revealed the monodispersity of particles and formation of 2D patterns. The space between the particles is around $3 \mathrm{~nm}$, corresponding to the thickness of two monolayers of oleic acid coating the particles (length of an oleic acid molecule is around $1.5 \mathrm{~nm}$ ). The number-based mean particle size on 300 counted particles of the sample S1 obtained from the TEM image is 8 $\mathrm{nm} \pm 3 \mathrm{~nm}$. The average crystallite size obtained from XRD data is 5.7(9) nm. 

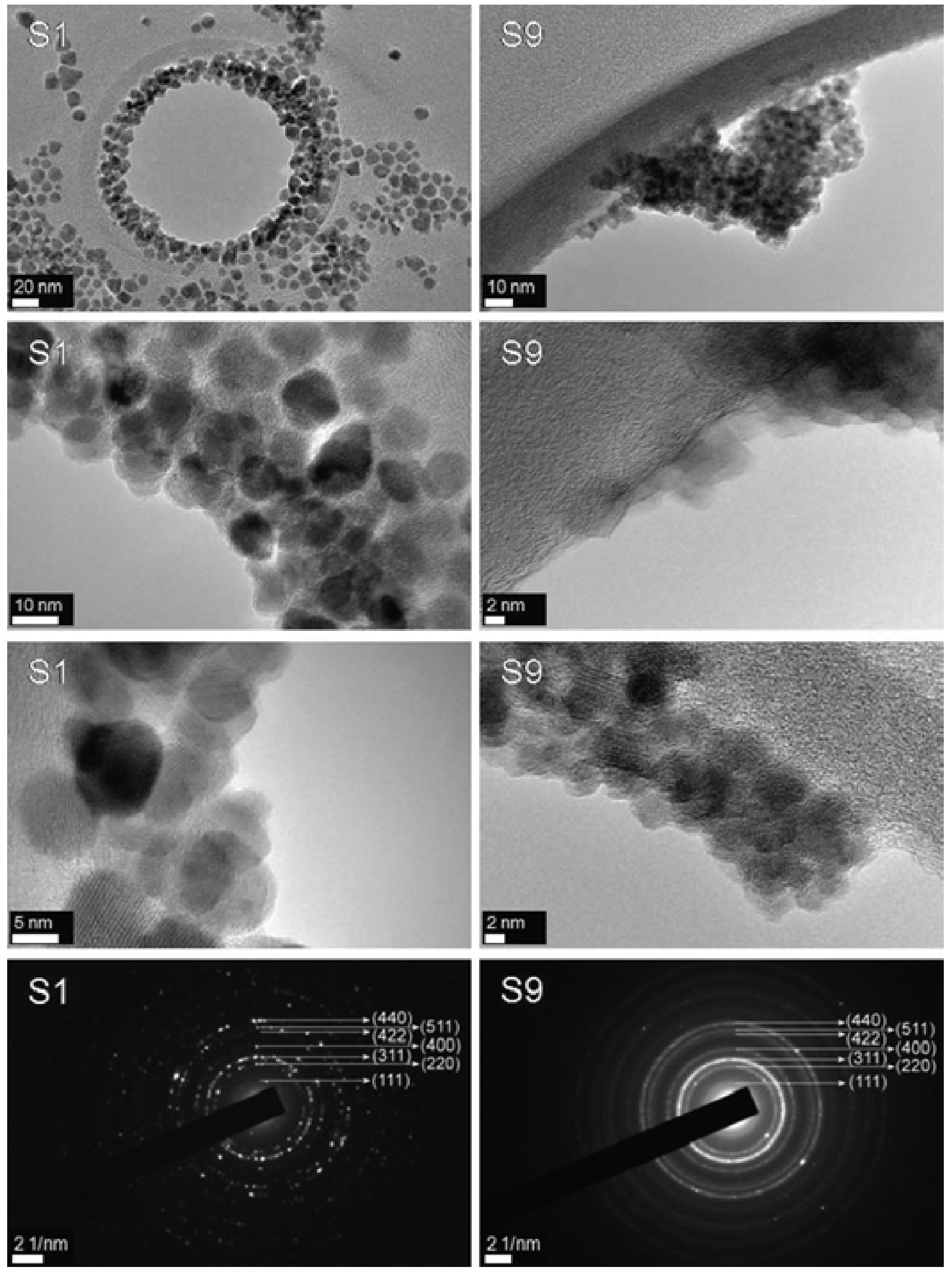

Figure 3. TEM images and SAED patterns of the sample S1, obtained from ferrous sulfate as the precursor salt, and S9, obtained from ferric chloride as the precursor salt. 
The sample S9 consists of extremely small cubic and rhombohedral crystals of around $5 \mathrm{~nm}$ in size. In the case of this sample, we have observed nanocrystals more uniform in size and shape, which most likely can be attributed to the solvolysis of the oleate complex in a homogenous medium, which indicates a transparent solvothermal precursor, as shown in Figure $1 \mathrm{~B}$, and a gentle heating process.

The volume-based particle size distribution of the sample S8 obtained by DLS is shown in Figure 4. The distribution of particles is rather narrow: 86 vol. \% of the particulate material have a mean hydrodynamic diameter of $27 \mathrm{~nm}$ with a standard deviation of $\pm 4 \mathrm{~nm}$. The presence of additional peaks, indicative of larger particles, can be attributed to the instability due to the fact that the ligand is simply desorbed in a bulk of the solvent [40]. The number-based mean particle size for this sample obtained from TEM images (340 counted particles) is $6 \pm 2 \mathrm{~nm}$. The average crystallite size calculated from XRD data is 5.6(9) nm. Furthermore, the disagreement with the TEM analysis regarding the particle sizes, which are smaller than $27 \mathrm{~nm}$, also suggests that particle aggregation occurred.
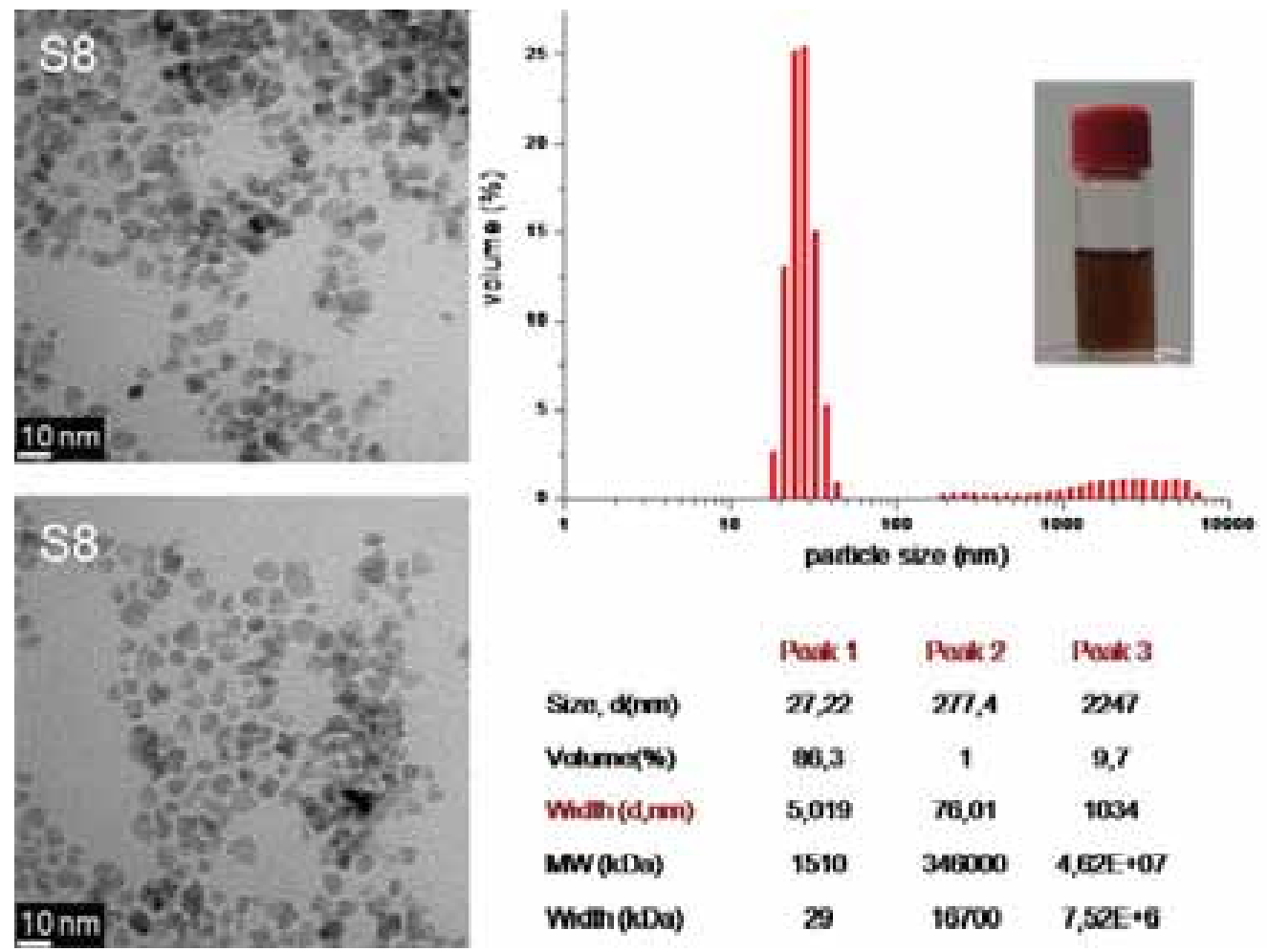

Figure 4. Representative TEM images of the sample S8. Particle size distribution and data obtained by DLS measurement of sample S8. DLS data are presented as mean values of three independent measurements (each with five replicates).

The magnetization curves of the samples S7 and S9 are shown in Figure 5 (blue dotted lines). The samples exhibit superparamagnetic behavior. The saturation magnetization of the samples is $51.52 \mathrm{emu} / \mathrm{g}$ and $40.42 \mathrm{emu} / \mathrm{g}$ at $14 \mathrm{kOe}$. The XRD analysis indicates that the sample S7 has a higher degree of crystal structure ordering and a larger crystallite size compared to S9. The crystallite sizes calculated from Sherrer's formula for the samples S7 and S9 are 6.1(2) $\mathrm{nm}$ and 5.2(2) nm, respectively. This could explain the larger saturation magnetization in the sample S7 since magnetic properties strongly correlate with the particle sizes and crystal structure [41].

The field-dependent magnetization results obtained from VSM at room temperature were fitted according to the Langevin equation, which is known to describe superparamagnetism of magnetic nanoparticles 
$[42,43,44,45]$. The fitted curves are shown in Figure 5 with a solid line. We have fitted this dependence to the modified Langevin function:

$$
\mathrm{M}=\mathrm{M}_{\mathrm{S}} \mathrm{L}\left(\mathrm{m}_{\mathrm{p}} \mathrm{H} / \mathrm{k}_{\mathrm{B}} \mathrm{T}\right)+\chi \mathrm{H}
$$

where $\mathrm{m}_{\mathrm{p}}$ is the magnetic moment of a particle, $\mathrm{k}_{\mathrm{B}}$ is the Boltzmann constant, $\mathrm{L}(\mathrm{x})=\operatorname{coth}(\mathrm{x})-1 / \mathrm{x}$ and $\chi$ is the high field susceptibility. Fitting was performed according to the least-square method in order to obtain the average magnetic moment of the particles. Assuming that the particles are spherical, the average particle diameter has been calculated according to the relationship:

$$
\mathrm{m}_{\mathrm{p}}=\mathrm{M}_{\mathrm{S}} \mathrm{V}
$$

where $\mathrm{M}_{\mathrm{S}}$ is the bulk saturation magnetization of magnetite and $\mathrm{V}$ is the volume of the particle. We determined by this method the sizes of magnetic particles: $4.3 \mathrm{~nm}$ and $4 \mathrm{~nm}$ for the samples $\mathrm{S} 7$ and $\mathrm{S} 9$, respectively. It can be observed that the calculated sizes of magnetic particles are very close to the crystal and particle sizes obtained from the XRD and TEM studies. These results also demonstrate that the main magnetic phase in our samples is magnetite.
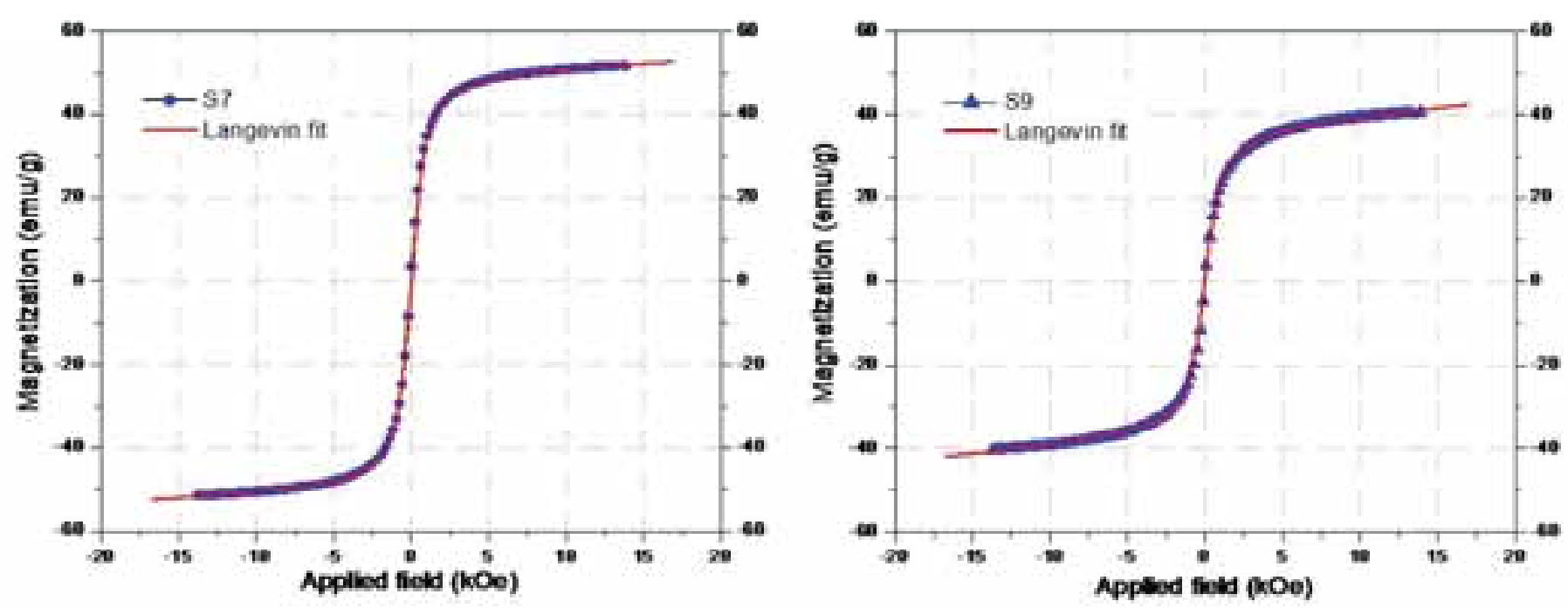

Figure 5. Field-dependent magnetization curves $(\mathrm{M}-\mathrm{H})$ (dots) at room temperature and the Langevine function fit (solid line) for the samples S7 and S9.

The TEM images presented in Figure 6 show that nanocrystals are well-dispersed, that they form planar structures on carbon support, and that a large number of particles are interconnected within the polymer matrix. In this specific sample, larger crystals identified as $\mathrm{NaCl}$ crystals have been detected. The particles are nonagglomerated, but are most likely organized in a 3D net pattern like polymeric aggregates [46, 47], with a relatively low volumetric density of nanocrystals compared to the polymer content. Figure $6 \mathrm{E}$ shows digital images of the test tubes containing the PLA-PEI/MNp dispersion stabilized with PVA. After one-month storage on $4{ }^{\circ} \mathrm{C}$, the dispersion was treated for $30 \mathrm{~min}$ in an ultrasonic bath and then the size distribution was measured (figure 6F). After a 30 min of treatment with low-intensity ultrasound, the following results were obtained: the particle size distribution was very narrow ( $\operatorname{span}=1.49$ ), indicating a high degree of uniformity, where $10 \%$ of particles had a diameter smaller than $\mathrm{d}(0.1)=35 \mathrm{~nm}, 50 \%$ of particles had a diameter of $\mathrm{d}(0.5)=66 \mathrm{~nm}$, while $90 \%$ of particles were smaller than $\mathrm{d}(0.9)=134 \mathrm{~nm}$ (figure $6 \mathrm{~F}$ ). 

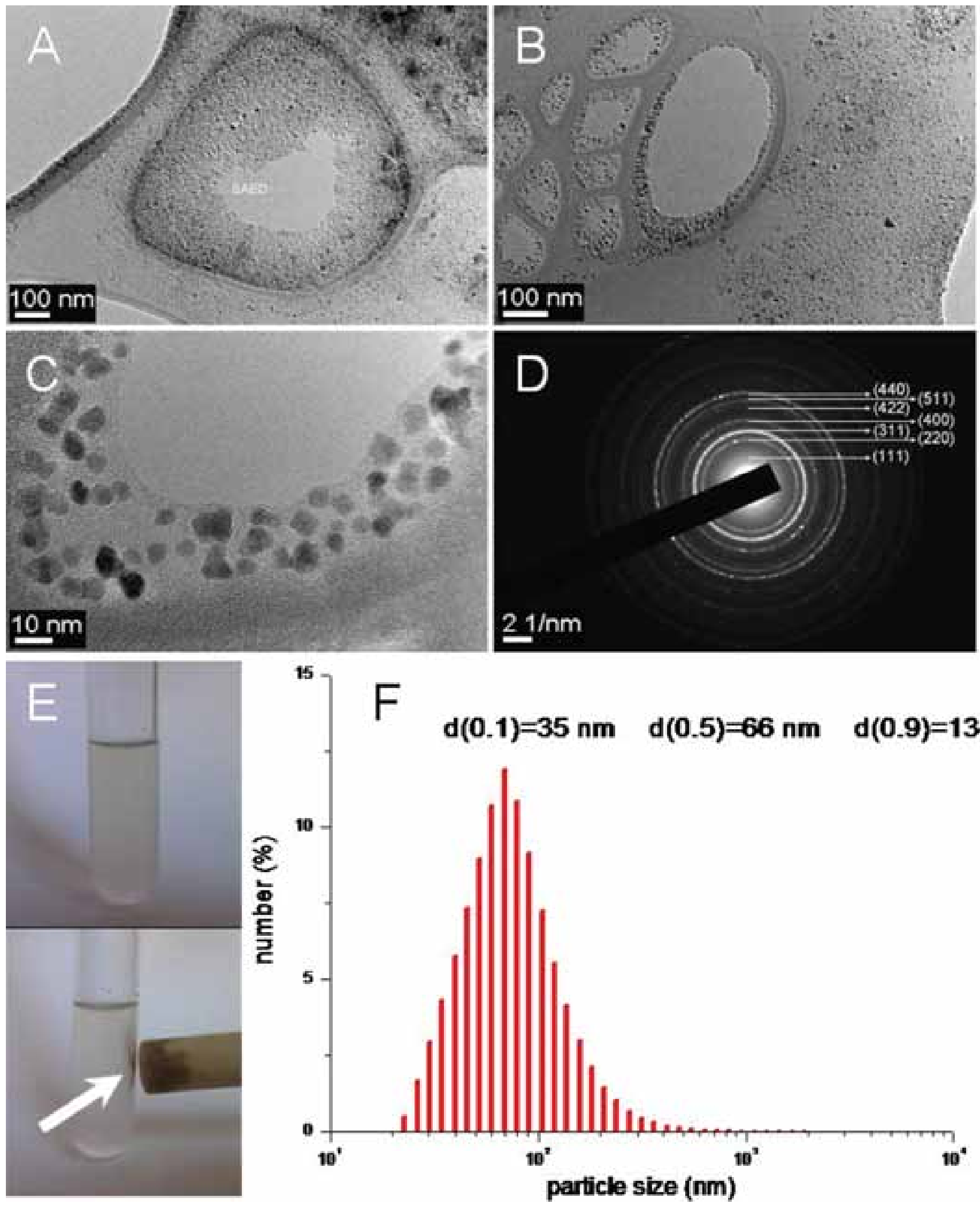

Figure 6. TEM images of magnetic nanocrystals immobilized within the poly-(L-lactide) polymer from water dispersions (A, B and C), and SAED of particles (D). The water dispersion of PLA-PEI/MNp stabilized with PVA attracted with a low energy magnet (E) and the size distribution of such hybrid particles measured immediately after sonication (F). 


\section{Conclusion}

In this study, we have presented in detail a simplified solvothermal process for the synthesis of highquality monodisperse magnetic iron oxide nanocrystals which employs glucose as a reducing agent and does not require a protective atmosphere or any complicated operations. We have also transformed hydrophobic nanocrystals into a water-soluble form using amphiphilic poly (L-lactide), which has a high hydrophobic load capacity, in combination with branched polyethyleneimine, a versatile functional polymer that could be used in gene transfection and/or cell adhesion applications. The reported nanoparticles have potential for biomedical applications, such as the magnetic separation of biomarkers or magnetic resonance imaging.

Acknowledgements. The authors would like to thank to MSc. Uroš Jovanović for DLS measurements and MSc. Sonja Jovanović for magnetic measurements. We also thank Dr. Smilja Marković for her help in interpreting the IR spectrum and Dr. Srečo D. Škapin for his help in characterizing the samples. The study has been carried out within the framework of the national project funded by the Serbian Ministry of Science, Education and Technological Development, Grant no. III 45004, and the Korean Institute of Science and Technology - Institute of Technical Science of SASA joint research project Advanced Materials for Biomedical Applications. The National Research Foundation of Korea (Grant Number R11-2008-0061852) is also acknowledged for support.

\section{References}

1. J.W.M. Bulte and D.L. Kraitchman, NMR Biomed. 17 (2004) 484;

2. C. Plank, U. Schillinger, F. Scherer, C. Bergemann, J.S. Rémy, F. Krötz, M. Anton, J. Lausier, J. Rosenecker, Biol. Chem. 384(5) (2003) 737;

3. J.T. Kemsheadl and J. Ugelstad, Mol. Cell. Biochem., 67 (1985) 11;

4. S. Miltenyi, W. Muller, W. Weichel, and A. Radbruch, Cytom. Part A 11(2) (1990) 231,

5. G.D. Chen, C.J. Alberts, W. Rodriguez, and M. Toner, Anal. Chem. (ACS) 82 (2010) 723;

6. A. Jordan, R. Scholz, P. Wust, H. Fahling and R. Felix, J. Magn. Magn. Mater. 201 (1999) 413;

7. Natalie A. Frey and Shouheng Sun, in C. Altavilla and E. Ciliberto (Eds.), Inorganic Nanoparticles: Synthesis, Applications, and Perspectives, CRC Press, Boca Raton FL, 2010, Chapter 3;

8. M.J. Jacinto, F.P. Silva, P.K. Kiyohara, R. Landers and L.M. Rossi, ChemCatChem 4(5) (2012) 698;

9. W. Yantasee, C.L. Warner, T. Sangvanich, R.S. Addleman, T.G. Carter, R.J. Wiacek, G.E. Fryxell, C. Timchalk and M.G. Warner, Environ. Sci. Technol. 41 (2007) 5114;

10. K. Raj, B. Moskowitz, R. Casciari, J. Magn. Magn. Mater. 149 (1995) 174;

11. B.J. Park, F.F. Fang and H.J. Choi, Soft Matter 6 (2010) 5246;

12. X. Wang and Y. Li, Chem. Commun. (Cambridge, U. K.) (2007) 28: 2901

13. J. Park, K. An, Y. Hwang, J.G. Park, H.J. Noh, J.Y. Kim, J.H. Park, N.M. Hwang and T. Hyeon, Nat. Mater. 3 (2004) 891; 
14. S. Sun and H. Zeng, J. Am. Chem. Soc. 124 (2002) 8204;

15. B.H. Kim, N. Lee, H. Kim, K. An, Y.I. Park, Y. Choi, K. Shin, Y. Lee, S.G. Kwon, H.B. Na, J.G. Park, T.Y. Ahn, Y.W. Kim, W.K. Moon, S.H. Choi and T. Hyeon, J. Am. Chem. Soc. 133 (2011) 12624;

16. C. Pereira, A.M. Pereira, C. Fernandes, M. Rocha, R. Mendes, M. Paz Fernández-García, A. Guedes, P.B. Tavares, J.M. Grenèche, J.P. Araújo and C. Freire, Chem. Mater. 24 (2012) 1496;

17. A.H. Lu, E.L. Salabas, and F. Schüth, Angew. Chem., Int. Ed. 46 (2007) 1222;

18. G. Demazeau, J. Mater. Sci. 43(7) (2008) 2104;

19. K. Byrappa and T. Adschiri, Prog. Cryst. Growth Charact. Mater. 53 (2007) 117;

20. B. Xu and X. Wang, Dalton Trans. 41 (2012) 4719;

21. X. Wang, J. Zhuang, Q. Peng and Y. Li, Nature 437 (2005) 121;

22. A. Repko, D. Nižňansky and J. Poltierová-Vejpravová, J. Nanopart. Res. 13 (2011) 5021;

23. J. Wang, J. Sun, Q. Sun, Q. Chen, Mater. Res. Bull. 38 (2003) 1113;

24. Y. Zheng, Y. Cheng, F. Bao, Y. Wang, Mater. Res. Bull. 41 ( 2006) 525;

25. TJ Daou, G Pourroy, S Bégin-Colin, JM Grenéche, C Ulhaq-Bouillet, P Legaré, Chem. Mater. 18 (2006) 4399 ;

26. S. Zhu, J. Guo, J. Dong, Z. Cui, T. Lu, C. Zhu, D. Zhang, J. Ma, Ultrasonics Sonochem. 20(3) (2013) 872;

27. X. Sun, C. Zheng, F. Zhang, Y. Yang, G. Wu, A. Yu and N. Guan, J. Phys. Chem. C 113 (2009) 16002;

28. L. Xiao, J. Li, D.F. Brougham, E.K. Fox, N. Feliu, A. Bushmelev, A. Schmidt, N. Mertens, F. Kiessling, M. Valldor, B. Fadeel, and S. Mathur, ACS Nano, 5(8) (2011) 6315;

29. B. Behdadfar, A. Kermanpur, H. Sadeghi-Aliabadi, M. del Puerto Morales, M. Mozaffari, J. Magn. Magn. Mater. 324 (2012) 2211;

30. W. Lu, Y. Shen, A. Xie and W. Zhang, J. Magn. Magn. Mater. 322 (2010) 1828;

31. M. Jin, G. He, H. Zhang, J. Zeng, Z. Xie and Y. Xia, Angew. Chem., Int. Ed. 50(45) (2011) 10560;

32. X. Wang, L. Zhou, Y. Ma, X. Li and H. Gu, Nano Res. 2(5) (2009) 365;

33. S. Palchoudhury, W. An, Y. Xu,Y. Qin, Z. Zhang, N. Chopra, R. A. Holler, C. Heath Turner, Y. Bao, Nano Lett. 11 (2011) 1141;

34. G. Salas, C. Casado, F. J. Teran, R. Miranda, C. J. Serna, M. Puerto Morales, J. Mater. Chem., 22 (2012) 21065;

35. R. J. Jandacek, Lipids 26(3) (1991) 250;

36. K. Yang, H. Peng, Y. Wen and N. Li, Appl. Surf. Sci. 256 (2010) 3093;

37. K. Prabu and E. Natarajan, Adv. Appl. Sci. Res., 3(2) (2012) 1870;

38. V. Tzitzios, G. Basina, M. Gjoka, V. Alexandrakis, V. Georgakilas, D. Niarchos, N. Boukos and D. Petridis, Nanotechnol. 17 (2006) 3750;

39. L.M. Bronstein, X. Huang, J. Retrum, A. Schmucker, M. Pink, B.D. Stein and B. Dragnea, Chem. Mater. 19 (2007) 3624;

40. E. Giovanelli, E. Muro, G. Sitbon, M. Hanafi, T. Pons, B. Dubertret and N. Lequeux, Langmuir 28(43) (2012) 15177;

41. G.F. Goya, T.S. Berquó, F.C. Fonseca and M.P. Morales, J. Appl. Phys. 94 (2003) 3520; 
42. M. Tadic, D. Markovic, V. Spasojevic, V. Kusigerski, M. Remskar, J. Pirnat, Z. Jaglicic, J. Alloys Compd. 441 (2007) 291;

43. M. Tadic, V. Kusigerski, D. Markovic, I. Milosevic, V. Spasojevic, J. Mag. Mag. Mater. 321 (2009) 12;

44. V. Kusigerski, M. Tadic, V. Spasojevic, B. Antic, D. Markovic, S. Boskovic, B. Matovic, Scr. Mater. 56 (2007) 883;

45. P. Dutta, A. Manivannan, M.S. Seehra, N. Shah, G.P. Huffman, Phys. Rev. B 70 (2004) 174428

46. A. Petri-Fink, B. Steitz, A. Finka, J. Salaklang, H. Hofmann, Eur. J. Pharm. Biopharm. 68 (2008) 129-137;

47. M. Cortia, A. Lascialfaria, M. Marinoneb, A. Masottid, E. Micottia, F. Orsinib, G. Ortaggid, G. Polettib, C. Innocentid and C. Sangregorioe, J. Magn. Magn. Mater. 320 (2008) e316. 